\title{
CONTINUA NOT AN INVERSE LIMIT WITH A SINGLE BONDING MAP ON A POLYHEDRON
}

\author{
J. W. ROGERS, JR.
}

1. Introduction. By a continuum here we mean a compact, connected metric space; by a polyhedron, a nondegenerate triangulable continuum. If $K$ is a continuum, let $L(K)$ denote the collection of all continua homeomorphic to a limit of an inverse sequence of mappings from $K$ onto $K$, let $S(K)$ denote the collection of all continua homeomorphic to a limit of an inverse sequence with a single bonding map on $K$, and let $I$ denote the interval $[0,1]$.

It is known ([4] or $\left[9\right.$, Theorem $\left.1^{*}\right]$, together with $[10$, Theorem 5]) that $L(I)$ is also the collection of all nondegenerate chainable continua, and the mappings involved in an inverse limit sequence for a chainable continuum are often thought of as following a pattern suggested by the successive chains for the continuum. Nevertheless, Henderson showed [5] that the pseudo-arc, in spite of its increasingly complex patterns of chaining, is an element of $S(I)$, while Mahavier [8] showed that there is another element of $L(I)$ which is not in $S(I)$. Mahavier's result raised the problem of characterizing the elements of $S(I)$, which is still unsettled (a partial solution can be found in [11]). Recently, Jolly and J. T. Rogers have shown [6] that there are four maps from $[0,1]$ into $[0,1]$ such that every chainable continuum can be obtained as an inverse limit using only these four maps.

In this note we generalize Mahavier's result by showing that if $P$ is any polyhedron, then $S(P)$ is a proper subcollection of $L(P)$. This is not the case if $P$ is any continuum, for if $P$ is a pseudo-arc then each element of $L(P)$ is a pseudo-arc, and $S(P)=L(P)$.

Theorem 1 seems of some interest in itself. Burgess has shown [2] that not every chainable continuum can be obtained as an inverse limit on circles. Curiously, this is a peculiarity of one-dimensional polyhedra, as the theorem shows (Fort and Segal have already shown [3] that an arc is obtainable as an inverse limit on 2-cells).

\section{Preliminaries.}

Definition. If $\epsilon>0$, a transformation $f$ from a metric space $X$ onto a space $Y$ is called an $\epsilon-m a p$ if and only if $f$ is continuous and if $P$ is a point of $Y$, then $f^{-1}(P)$ has diameter $\leqq \epsilon$. The space $X$ is said to be $Y$-like if and only if there is an $\epsilon$-map from $X$ onto $Y$ for each $\epsilon>0$ [9]. 1968.

Presented to the Society, November 9, 1968; received by the editors August 5 , 
THEOREM 1. If $n>1, P$ is an $n$-dimensional polyhedron, and $C$ is a chainable continuum, then $C$ is an element of $L(P)$.

Proof. By a result of Kuratowski and Ulam [7, p. 251], if $n>1$ and $P$ is an $n$-dimensional polyhedron, then the interval $[0,1]$ is $P$-like. Also, since $C$ is chainable, $C$ can be $\epsilon$-mapped onto $[0,1]$ for each $\epsilon>0$. Hence $C$ is $P$-like, and by a theorem of Mardešic and Segal [9, Theorem 1, p. 148], $C$ is an element of $L(P)$.

TheOREM 2. If $P$ is a one-dimensional polyhedron, there is an element $M$ of $L(P)$ such that the only homeomorphism from $M$ onto $M$ is the identity.

Proof. Let $K$ denote a triangulation of $P, s_{1}, \cdots, s_{n}$ denote the one-simplexes of $K$, and $M$ denote the chainable continuum of Andrews [1], no two of whose nondegenerate subcontinua are homeomorphic. $M$ contains $n$ mutually exclusive continua, $M_{1}, \cdots, M_{n}$, such that for each $i(1 \leqq i \leqq n) M_{i}$ is chainable from a point $A_{i}$ to another point $B_{i}$. Let $T$ denote a transformation that throws, for each $i(1 \leqq i \leqq n), A_{i}$ onto one endpoint of $s_{i}$ and $B_{i}$ onto the other. Let $M^{\prime}$ denote the continuum obtained from $M_{1}, \cdots, M_{n}$ by identifying points that are thrown by $T$ on to the same vertex of $K$. Clearly then $M^{\prime}$ is an element of $L(P)$. Moreover, if $\varnothing$ is a nontrivial homeomorphism from $M^{\prime}$ onto $M^{\prime}$, then some two nondegenerate subcontinua of $M^{\prime}$ are homeomorphic, which is impossible.

3. Limits with single bonding maps. Little change is required in Mahavier's proof of his Theorem 1 [8] to show:

TheOREM 3. If $P$ is a polyhedron and $M$ is an element of $S(P)$, then there is a nontrivial homeomorphism from $M$ onto $M$.

THEOREM 4. If $P$ is a polyhedron, there exists an element of $L(P)$ which is not in $S(P)$.

Proof. If $P$ is one-dimensional, then Theorems 2 and 3 give the conclusion. If $P$ is $n$-dimensional $(n>1)$, then by Theorem 1 every chainable continuum is an element of $L(P)$, and again Theorems 2 and 3 suffice.

\section{REFERENCES}

1. J. J. Andrews, $A$ chainable continuum no two of whose nondegenerate subcontinua are homeomorphic, Proc. Amer. Math. Soc. 12 (1961), 333-334.

2. C. E. Burgess, Chainable continua and indecomposability, Pacific J. Math. 9 (1959), 653-659. 
3. M. K. Fort, Jr. and J. Segal, Local connectedness of inverse limit spaces, Duke Math. J. 28 (1961), 253-260.

4. H. Freudenthal, Entwicklungen von Rüumen und ihren Gruppen, Compositio Math. 4 (1937), 145-234.

5. G. W. Henderson, The pseudo-arc as an inverse limit with one binding map, Duke Math. J. 31 (1964), 421-425.

6. R. F. Jolly and J. T. Rogers, Jr., Inverse limit spaces defined by only finitely many distinct bonding maps, Fund. Math. (to appear).

7. C. Kuratowski and S. Ulam, Sur un coefficient lie aux transformations continues d'ensembles, Fund. Math. 20 (1933), 244-253.

8. W. S. Mahavier, A chainable continuum not homeomorphic to an inverse limit on $[0,1]$ with only one bonding map, Proc. Amer. Math. Soc. 18 (1967), 284-286.

9. S. Mardesic and J. Segal, $\in$-mappings onto polyhedra, Trans. Amer. Math. Soc. 109 (1963), 146-164.

10. M. C. McCord, Universal P.like compacta, Michigan Math. J. 13 (1966) 71-85.

11. J. W. Rogers, Jr., Decomposable inverse limits with a single bonding map on $[0,1]$ below the identity, Fund. Math. (to appear).

\section{EMORY UNIVERSITY}

